\title{
From Flamsteed to Piazzi and Lalande: new standards in 18th century astrometry ${ }^{\star}$
}

\begin{abstract}
James Lequeux
LERMA, Observatoire de Paris, 61 Av. de l'Observatoire, 75014 Paris, France

e-mail: james.lequeux@obspm.fr

Received 28 March 2014 / Accepted 18 May 2014

\section{ABSTRACT}

Aims. The present high accuracy of stellar positions and proper motions allows us to determine the positional accuracy of old stellar catalogues. This has already been done for the most important catalogues from before the 18th century. Our aim is to extend this study to several 18 th century catalogues.

Methods. To do this, I studied ten catalogues: those of Flamsteed and Rømer, four catalogues of La Caille, and catalogues of Tobias Mayer, Bradley, Piazzi, and Lalande. A comparison with modern data, mostly from HIPPARCos, compiled in the Simbad database of the CDS allowed me to determine the position errors of these catalogues. I also compared the stellar visual magnitudes given in eight of these catalogues with photometric $V$ magnitudes.

Results. Thanks to novel instruments, the rms positional accuracy improved from thousands to hundreds of arcsec in older catalogues to less than one minute in that of Flamsteed, and to 2-6 arcsec in the other catalogues I examined. These improvements allowed for the first time relatively accurate proper motions to be determined by 19 th century astronomers.
\end{abstract}

Key words. history and philosophy of astronomy - astrometry - catalogs

\section{Introduction}

The evolution of accuracy in the determination of stellar positions from antiquity to the present time has been the subject of several studies, for example by $\mathrm{Høg}(2008,2009)$ and references therein. Detailed studies have recently been published on the accuracy of ancient stellar catalogues: Ptolemaios and Ulugh Beg (Verbunt \& van Gent 2012), Tycho Brahe (Verbunt \& van Gent 2010a), early catalogues of the Southern sky (Verbunt \& van Gent 2011), and the catalogue of Hevelius (Verbunt \& van Gent 2010b). There is a lack of such studies for later catalogues. This is a gap that I aim at filling for the catalogue of Flamsteed and for a large selection of catalogues of the 18th century.

The only correction made by the authors of the catalogues studied by Verbunt and van Gent and by Flamsteed was for precession. Aberration and nutation were unknown from them, as they were only discovered by Bradley and published by him in 1727 and 1748, respectively. Because the date of the observation is required to reduce the observed stellar position to the mean position when taking into account aberration and nutation, it is impossible to include them in the reduction of old catalogues that do not indicate these dates. An exception is the catalogue of Flamsteed, for which the dates of the observations are specified. As a consequence, the accuracy of the observations themselves is somewhat underestimated by these incomplete reductions, but not by very much, because the accuracy of the measurements is poor. This is no longer the case for the other catalogues I examine here.

* The catalogues with some corrections are available in digital form at the CDS via anonymous ftp to cdsarc.u-strasbg. fr $(130.79 .128 .5)$ or via

http://cdsarc.u-strasbg.fr/viz-bin/qcat?]/A+A/567/A26
My analysis is based on modern data on positions and proper motions gathered in the Simbad database of the Centre de Données de Strasbourg (CDS). Most of these data come from the HIPPARCos catalogue. The position errors in the database are completely negligible, and the errors on the proper motions are so small that they correspond most often to less than one arc second over the intervals between the observations and J2000. I treat separately the right ascensions and the declinations in each catalogue. I first examine the catalogue of Flamsteed, then the small stellar catalogue of Rømer, several catalogs produced by La Caille, and finally the catalogues of Tobias Mayer, Bradley, Piazzi, and Lalande. This is an arbitrary choice as there are other 18th century catalogues of comparable accuracy, for instance that of Maskelyne. However, this catalogue was obtained with the same instrument as that of Bradley and should be of comparable accuracy.

\section{Method}

For convenience, I precessed the mean equatorial coordinates (or, for Flamsteed, the ecliptic coordinates) given in the catalogues to J2000 equatorial coordinates using the on-line NED calculator $^{1}$, then I searched for an identification around this position in Simbad. This allowed nearly always a secure identification, without ambiguity. When this was not possible, I used the original designation of the star given by the author to obtain the corresponding Simbad position; this allowed me to discover some obvious clerical errors that I corrected in the original. Some entries could not be identified at all.

Then I applied the proper motions in right ascension and declination to the (sometimes corrected) equatorial coordinates precessed to J2000, and subtracted the results from the coordinates

1 http://ned.ipac.caltech.edu/ 
Table 1. Position errors in ten 18th-century catalogues.

\begin{tabular}{|c|c|c|c|c|c|c|}
\hline Catalogue & $\begin{array}{r}\text { Number of stars } \\
\text { studied }\end{array}$ & $\begin{array}{l}\text { Number } \\
\text { retained }\end{array}$ & Mean & rms & St. dev. & $\begin{array}{l}\text { Internal error } \\
\text { (rms or st.dev) }\end{array}$ \\
\hline Flamsteed & 220 & RA: 195 & $-7.0^{\prime \prime}$ & $48.2^{\prime \prime}$ & $47.8^{\prime \prime}$ & $\approx 40^{\prime \prime}$ \\
\hline (from Lalande 1783) & (over 3925) & Dec: 195 & $-1.9^{\prime \prime}$ & $31.3^{\prime \prime}$ & $31.3^{\prime \prime}$ & $\approx 25^{\prime \prime}$ \\
\hline Rømer & 88 & RA: 88 & $-2.5^{\prime \prime}$ & $5.2^{\prime \prime}$ & $4.3^{\prime \prime}$ & $4.0^{\prime \prime}$ \\
\hline (from Galle 1845) & & Dec: 81 & $7.8^{\prime \prime}$ & $14.2^{\prime \prime}$ & $11.9^{\prime \prime}$ & $13^{\prime \prime}$ \\
\hline La Caille, fundamental stars & 398 & RA: 388 & $1.2^{\prime \prime}$ & $6.4^{\prime \prime}$ & $6.3^{\prime \prime}$ & $5.0^{\prime \prime}$ \\
\hline (La Caille 1757) & & Dec: 388 & $2.0^{\prime \prime}$ & $4.4^{\prime \prime}$ & $4.0^{\prime \prime}$ & $3.9^{\prime \prime}$ \\
\hline La Caille/Bailly, fundamental stars & 307 & RA: 298 & $0.3^{\prime \prime}$ & $5.9^{\prime \prime}$ & $5.9^{\prime \prime}$ & $5.7^{\prime \prime}$ \\
\hline (Anonymous (Bailly) 1763) & & Dec: 298 & $0.0^{\prime \prime}$ & $4.6^{\prime \prime}$ & $4.6^{\prime \prime}$ & $4.6^{\prime \prime}$ \\
\hline La Caille, southern survey & 244 & RA: 228 & $10.3^{\prime \prime}$ & $22.9^{\prime \prime}$ & $20.5^{\prime \prime}$ & $22^{\prime \prime}$ \\
\hline (La Caille 1847) & (over 9766) & Dec: 228 & $5.8^{\prime \prime}$ & $39.2^{\prime \prime}$ & $38.9^{\prime \prime}$ & $39^{\prime \prime}$ \\
\hline La Caille, zodiacal stars & 515 & RA: 487 & $1.2^{\prime \prime}$ & $5.6^{\prime \prime}$ & $5.6^{\prime \prime}$ & $4.2^{\prime \prime}$ \\
\hline (Anonymous (Bailly) 1763) & & Dec: 487 & $3.0^{\prime \prime}$ & $6.5^{\prime \prime}$ & $5.8^{\prime \prime}$ & $5.6^{\prime \prime}$ \\
\hline Mayer, zodiacal stars & 200 & RA: 195 & $3.3^{\prime \prime}$ & $5.9^{\prime \prime}$ & $4.9^{\prime \prime}$ & $3.7^{\prime \prime}$ \\
\hline (from Baily 1831) & (over 998) & Dec: 195 & $4.4^{\prime \prime}$ & $5.7^{\prime \prime}$ & $3.7^{\prime \prime}$ & $3.4^{\prime \prime}$ \\
\hline Bradley & 215 & RA: 212 & $1.4^{\prime \prime}$ & $3.1^{\prime \prime}$ & $2.8^{\prime \prime}$ & $3.0^{\prime \prime}$ \\
\hline (from Bessel 1818) & (over 3220) & Dec: 212 & $0.3^{\prime \prime}$ & $1.8^{\prime \prime}$ & $1.8^{\prime \prime}$ & $1.8^{\prime \prime}$ \\
\hline Piazzi & 203 & RA: 202 & $-0.7^{\prime \prime}$ & $3.2^{\prime \prime}$ & $3.1^{\prime \prime}$ & $3.2^{\prime \prime}$ \\
\hline (Piazzi 1814) & (over 7646) & Dec: 202 & $1.5^{\prime \prime}$ & $2.4^{\prime \prime}$ & $1.9^{\prime \prime}$ & $1.8^{\prime \prime}$ \\
\hline Lalande & 198 & RA: 188 & $-0.2^{\prime \prime}$ & $4.3^{\prime \prime}$ & $4.3^{\prime \prime}$ & $4.3^{\prime \prime}$ \\
\hline (from Bossert 1892) & (over $\approx 45000)$ & Dec: 188 & $1.5^{\prime \prime}$ & $4.1^{\prime \prime}$ & $3.8^{\prime \prime}$ & $4.0^{\prime \prime}$ \\
\hline
\end{tabular}

Notes. Errors are given as catalogue position minus modern position. Proper motion is accounted for.

given by Simbad to obtain the errors in right ascension and declination. As remarked by the referee, this method has the inconvenience that these errors are not in the original equatorial system but in the $\mathrm{J} 2000$ system, which is slightly rotated with respect to the original. The NED calculator gives the angle of rotation, which is fortunately always very small, except for a few stars near the poles: for example, it is smaller than 2.7 degrees for all the stars in the catalogue of Rømer, with the exception of the Pole star. Thus this should not affect our results appreciably. Of course, the total error is not affected by the rotation.

The catalogues with some corrections are available in digital form at the CDS: a random selection for those of Flamsteed, La Caille's southern survey, Mayer, Bradley, Piazzi and Lalande, in full for Rømer and the other catalogues of La Caille and Bailly. The designation of the stars in these electronic versions is not always the original one to make it compatible with entries in the Simbad database.

\section{Catalogue of Flamsteed}

John Flamsteed (1646-1719) was the first astronomer in charge of Greenwich Observatory. His stellar catalogue (Flamsteed 1725) was built on observations from 1675 to 1683 with a 6 -feet radius sextant mounted on an axis parallel to the polar axis of the Earth, then from 1683 to 1719 with a mural circle with a radius of 79.5 inches $(2 \mathrm{~m})$. Both instruments have disappeared. Flamsteed introduced in his catalogue the designation of stars of each constellation by numbers in order of increasing right ascension, a system corrected and improved by Jérôme Lalande (1732-1807). The ecliptic coordinates for epoch B1690 given in this catalogue are reproduced faithfully by Lalande (1783) with a number of corrections and name changes. Note that there are a number of missing or duplicate stars in the original catalogue, or stars for which the constellation has changed (Wagman 1987). This is another reason why I used Lalande's version. Lalande claims that the astronomers of his time were still using the original Flamsteed catalogue extensively: this why I included it in the present study and did not attempt to correct it for aberration and nutation, which would have required a considerable amount of work.

The catalogue contains 2925 stars in Lalande's version. Given this large number, I randomly extracted 220 stars from the catalogue by retaining one star for every 20 in the list. I converted their mean ecliptic coordinates given for B1690.0 into J2000 equatorial coordinates, and added the proper motions from Simbad, calculated between 1690 and 2000. Because the dates of observations differ from 1690, this introduces some errors for a few stars stars with large proper motions, but these errors are much smaller than the errors in the measurements. I corrected obvious typographical errors for three stars. I was unable to find seven stars close to the positions given by Flamsteed. Eighteen stars had position errors larger than 3 arcmin in at least one equatorial coordinate, and I decided to exclude them from the statistics. As a consequence, I was left with 195 stars for the statistical analysis.

The results of this analysis are reported in Table 1 and Fig. 1. Table 1 gives the mean error, the root mean square (rms) deviation around this mean, and the standard deviation, which should be equal for a Gaussian distribution. Figure 1 shows the individual errors in right ascension and declination as a function of declination, and the corresponding histograms. There is an offset of 7 arcsec in right ascension and a smaller one in declination. There is no clear systematic effect either with right ascension or declination. The rms deviation and standard deviation are 48 arcsec in right ascension and 31 arcsec in declination. The actual measurement accuracies are somewhat better because aberration and nutation are not taken into account: my estimates for these accuracies, which should be close to the internal errors of the observations, are 40 arcsec in right ascension and $25 \mathrm{~s}$ in declination. I conclude that the accuracy of Flamsteed's observations is 3-4 times better than those of Hevelius, which are somewhat earlier (Verbunt \& van Gent 2010b), which is essentially due to the use of a telescope, while Hevelius worked with the naked eye. 

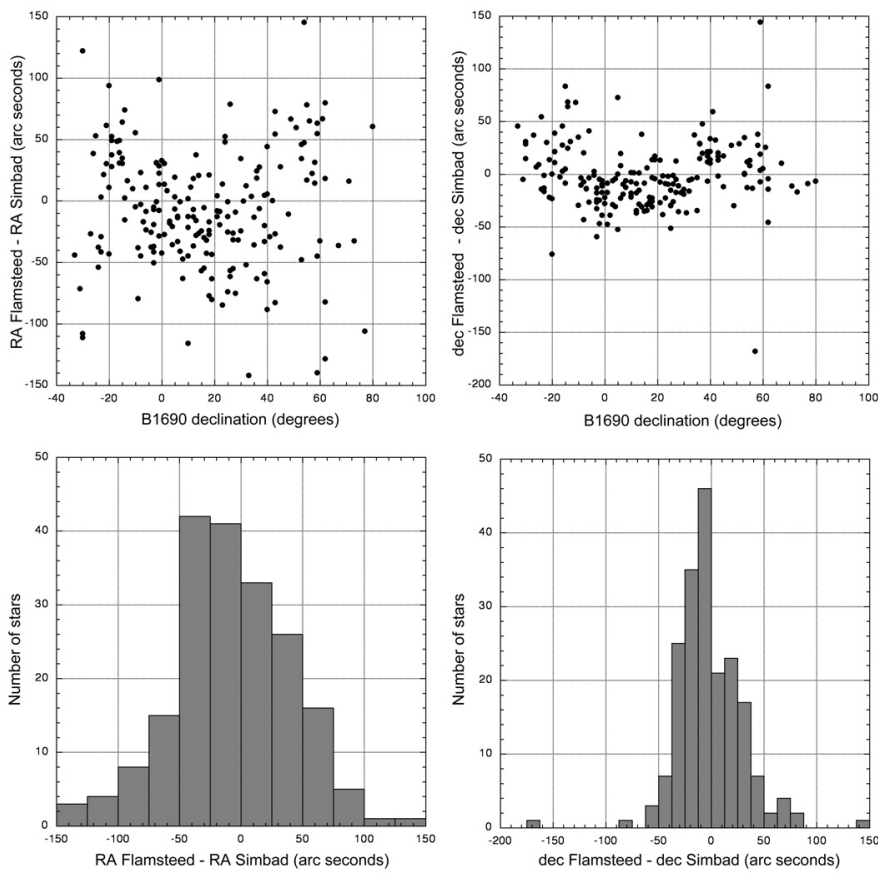

Fig. 1. Results of Flamsteed's measurements of 195 stars around 1690. Top left, errors in right ascension. Bottom left: histogram of errors in right ascension. Top right: errors in declination. Bottom right: histogram of errors in declination.

\section{Catalogue of Rømer}

Ole (or Olaus) Rømer (1644-1710) is principally known for his 1676 discovery of the finite velocity of light, a discovery that he shared with Jean-Dominique Cassini (Bobis \& Lequeux 2008). After a long stay in Paris, he returned to Copenhagen in 1681 and was appointed professor of astronomy at the University. He invented the meridian telescope that he installed in 1690 at a window of his house, as depicted in a famous engraving. He was also active in the nearby observatory at Rundetårn, where he installed a meridian telescope (the same or another one?), now with a vertical circle of 5 1/4 feet diameter. The observatory and all the observations were destroyed in the great Copenhagen fire of 1728, with the exception of observations of 88 stars obtained during three observing nights, from 20 to 23 October 1706. They were made with the second instrument. As the dates of the observations are known, it is possible to reduce them including aberration and nutation. This was done by Johann Gottfried Galle (1812-1910), the astronomer who first saw Neptune in 1846. The reduction of Rømer's observations was the subject of his doctoral thesis (Galle 1845). He had to use his own table for refraction and to guess the temperature during Rømer's observations.

I was easily able to identify all stars of this small catalogue. All 88 have measured right ascensions, but only 81 have measured declinations. To compare their mean positions as given by Galle for equinox B1706.0 with the Simbad positions, I precessed them to J2000.0 using the NED calculator and added the proper motions from Simbad. The result of my analysis is reported on Fig. 2 and Table 1. The errors in right ascension show a marginal systematic effect with declination, so I also give in Table 1 the rms of the errors with a correction for this effect using the linear fit shown in Fig. 2: this should correspond to the internal error of the observations.
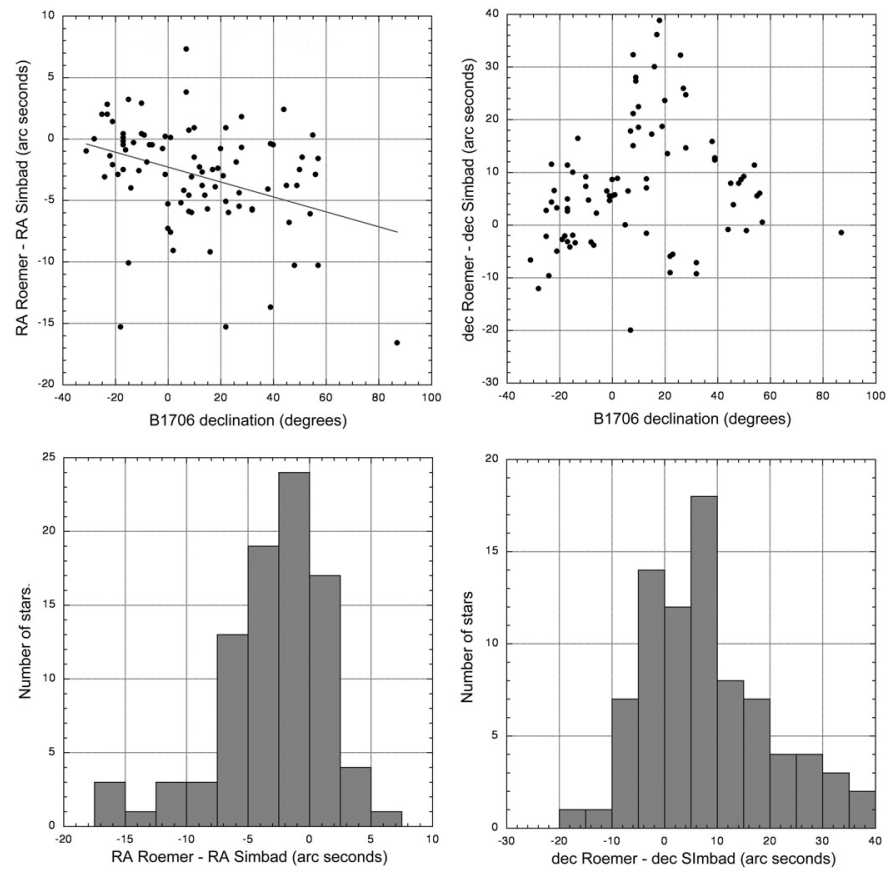

Fig. 2. Results of Rømer's measurements of 88 stars in 1706. Top left, errors in right ascension as a function of declination, with a linear fit. Bottom left: histogram of errors in right ascension. Top right: errors in declination as a function of declination for 81 stars. Bottom right: histogram of errors in declination.

The accuracy of the measurements in right ascension (5" rms, 4" after correction) is remarkable, especially given that there was probably only one single measurement for each star. This shows the skill of the observer and the considerable improvement allowed by Rømer's new meridian telescope compared with the instruments used by others at the same epoch, those of Flamsteed in particular. The accuracy of declination (14" rms) is poor, however. This cannot be due to the refraction table of Galle, which is quite correct, but more probably to the instrument.

\section{La Caille's catalogue of fundamental stars}

Nicolas-Louis La Caille (or Lacaille, or de la Caille, 1713-1762) was a French astronomer who is remembered principally for his survey of the southern sky, where he introduced 14 new constellations that are still in use today (see Glass 2013). Before leaving for the Cape of Good Hope in 1750, he started a catalogue of the 400 brightest stars of both hemispheres, which he completed during his stays in Cape Town and in the Mauritius island, then after his return to Paris in 1754 . He reduced the observations himself, including for the first time corrections for aberration and nutation, and published them with details of the observations and reductions (La Caille 1757). The way the observations were made is described by his pupil Jean-Sylvain Bailly (1736-1793), page lxxviii of the Ephemerides for 10 years from 1765 to 1775 (Anonymous (Bailly) 1763), as follows (my translation from French):

"The right ascensions of the stars were determined by a large number of corresponding heights of each of them taken with a quadrant (quart-de-cercle) of three feet radius to obtain their meridian passage with the greatest accuracy. For almost all stars 
of the northern hemisphere the right ascensions are relative to that of $\alpha$ Lyrae, and for the southern ones to that of Sirius, that is to say, each time the time of the meridian passage of a star has been found by corresponding heights, that of $\alpha$ Lyrae or of Sirius has been determined in the same way. The right ascensions of these two stars have been determined by a large number of observations made especially in the best possible opportunities and with the most exact methods known. The declinations have been derived from a large number of zenith distances observed with an instrument with a radius of six feet made especially with the utmost possible care".

The "method of corresponding heights" consisted of measuring the time of the passage of the observed star to the same zenith distance on each side of its meridian passage, then to take the mean of the two times. For this, La Caille used his 3-feet radius quadrant from 1746 to 1750 in Paris, then in 1751 and 1752 in Cape Town. For declinations, he used a 6-feet sextant made by Langlois, first in Paris in 1749-1750, then in Cape Town in 1751-52, in the Mauritius island in 1753 and again in Paris in 1755-1756. This instrument is preserved at the Paris Observatory.

La Caille's results are given p. 233 to 237 of his book (La Caille 1757). The final catalogue has 398 entries. I precessed the positions from B1750 to J2000 and added the proper motions in the same time interval of 250 years, for simplification. This might have introduced an error of 1 or 2 arcsec for a few stars with large proper motions, but several of these stars, such as $\alpha$ Centauri, have been observed at several epochs and La Caille only gives a mean position that ignores proper motion. I was able to identify all stars except the one in the list that precedes $\alpha$ Centauri, which corresponds certainly to some sort of confusion with artefacts from the images of this very bright star. I corrected the measurements for obvious typographic errors for two stars, which add to two other stars that were corrected by some hand in the copy scanned by Google books. For nine stars, the errors in right ascension or in declination are anomalously large and they were excluded from the statistics. Overall, I compiled a list of 388 stars from a total of 398 entries and 397 stars.

The results of my investigation are given in Table 1 and in Fig. 3. There is a clear systematic effect with declination in the right ascensions determined by La Caille. This is probably due to his constant reference to Sirius and Vega. The errors in right ascension for these two stars are 1.9 and -4.6 arcsec, their respective declinations being -16 and +38 degrees: they match the linear fit shown in Fig. 3 very well. However, this fit itself is arbitrary: it can be replaced by a simple offset for the northern stars and by another one for the southern stars. When corrected for the systematic deviation by using the linear fit, the rms error or its standard deviation in right ascension are reduced from 6.3 arcsec to 5 arcsec, which I expect is close to the internal error. The determination of declinations is slightly better, about 4 arcsec rms, with a rather marginal systematic effect with declination. When corrected for it by using the linear fit shown in Fig. 3, the rms error is reduced to 3.9 arcsec, which represents the internal error. The smallness of the systematic effect in declination is somewhat surprising because the refraction tables built by La Caille overestimate the refraction for stars low on the horizon. Maskelyne carefully analysed the problem and concluded that this overestimate was due to small inaccuracies in the sextant (Maskelyne 1787 , see p. 170-4); this was verified afterwards by Delambre (Delambre 1827, see p. 480-90). Maskelyne observed, however, that the fact that La Caille used the same instrument to determine the refractions and declinations of the bright stars minimized the errors due to refraction.
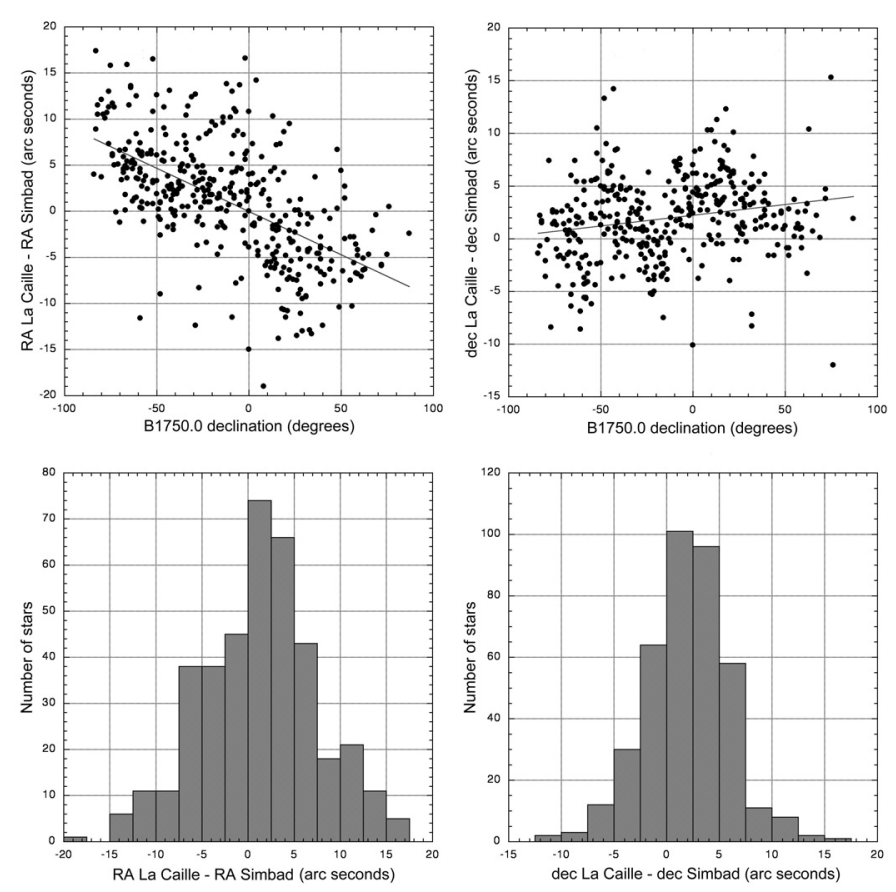

Fig. 3. Results of La Caille's measurements of 397 fundamental stars around 1750. Top left: errors in right ascension as a function of declination, with a linear fit. Bottom left: histogram of errors in right ascension. Top right: errors in declination as a function of declination, with a linear fit. Bottom right: histogram of errors in declination.

\section{Bailly's adaptation of La Caille's catalogue of fundamental stars}

After the death of La Caille, Jean-Sylvain Bailly published a catalogue of the brighest stars of both hemispheres for the equinox B1750.0 in Ephemerides for 10 year from 1765 to 1775 (Anonymous (Bailly) 1763, p. lvii-lxiv). This catalogue obviously derives from the catalogue described in the previous section. There are 303 stars in common, plus 4 added by Bailly, but the positions are slightly different, showing that Bailly made new reductions of La Caille's observations. I corrected obvious typographic or transcription errors for twelve stars and eliminated nine stars for which the errors in right ascension or in declination are anomalously large. I precessed the observed mean positions and corrected them for proper motion as for La Caille's catalogue.

The results are given in Table 1 . There is a marginal systematic effect with declination for the right ascensions, considerably lower than in La Caille's catalogue. I corrected for this effect using a linear fit of the errors in right ascension; the uncorrected and corrected results are given in Table 1. Apart for this systematic effect, the final quality is not better than that of La Caille's original catalogue.

\section{La Caille's complete survey of the southern sky}

During his stay in Cape Town in 1751-1752, La Caille made the first systematic survey of the sky ever, in the modern sense. He used a small telescope attached to the telescope of his 3-ft radius quadrant, oriented in the plane of the meridian. This telescope had a field of view of 3 degrees and had a special rhomboidal micrometer inside its eyepiece. La Caille oriented his telescope to some zenith distance for the whole night, measured the time of meridian crossing of every bright star, and obtained its zenith 

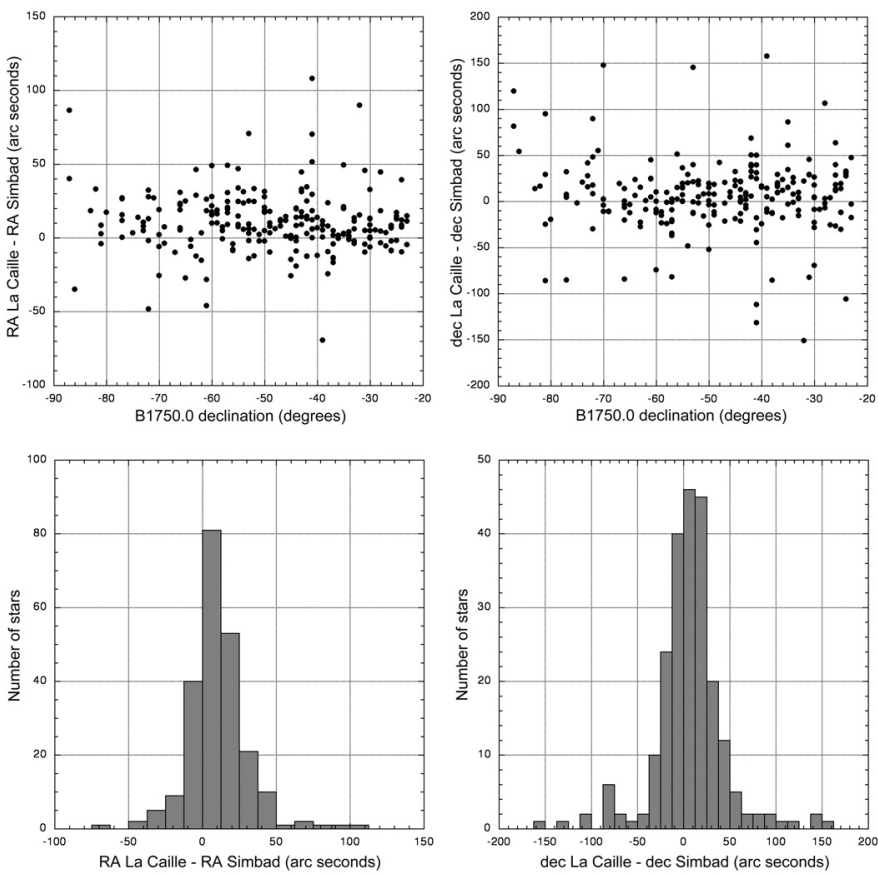

Fig. 4. Results of La Caille's measurements of a random sample of 228 of the 9766 stars of his southern survey. Top left: errors in right ascension as a function of declination. Bottom left: histogram of errors in right ascension. Top right: errors in declination as a function of declination. Bottom right: histogram of errors in declination.

distance relative to the field center from the duration of its passage through the micrometer. When the declination band was complete, he moved the telescope to another elevation. It took him 17 full nights and 110 additional sessions of eight hours each to cover the entire sky south of the tropic of Capricorn. He was alone to observe, to read the clocks, and to take notes. The full catalogue contains 9766 stars. La Caille had time to reduce only 1942 stars, and a complete reduction was made much later by Thomas Henderson (1798-1844) and Francis Baily (1774-1844), at the expense of the British Association for the Advancement of Science (La Caille et al. 1847). They probably hoped to obtain proper motions by comparison of the catalogue with that of John Herschel. Gould (1890a,b,c,d) noticed and corrected when possible a fair number of errors in the new catalogue. I chose to work only on a random sample of 244 stars, one for every 40 stars of the catalogue. No star could be identified for nine entries, and seven stars have very poor positions and were eliminated from the statistics.

The results for the remaining 228 stars are given in Fig. 4 and Table 1 . There is a systematic error of 10 arcsec in RA and 6 arcsec in DEC, but no noticeable variation with declination. The rms errors are 23 arcsec in right ascension and 39 arcsec in declination. This is consistent with the errors of $30 \operatorname{arcsec}$ estimated by La Caille himself.

\section{La Caille's catalogue of zodiacal stars}

When La Caille returned from his southern expedition in 1754, he undertook the construction of a catalogue of zodiacal stars, in order to obtain reference positions for the motion of planets. His purpose was to include 800 stars, but he was only able to observe 515 stars between September 1760 and his premature death in March 1762. They were reduced to B1765.0 by Jean-Sylvain Bailly and published by him together with his new reduction of
Table 2. Unidentified entries in La Caille's and Mayer's zodiacal catalogues.

\begin{tabular}{rrrrl}
\hline \hline La Caille & & & & \\
Cat. numb. & RA $(\mathrm{B} 1765.0)$ & Dec $(\mathrm{B} 1765.0)$ & Magnitude & Remarks \\
\hline 29 & $21 \mathrm{~d} 39 \mathrm{~m} 18.6 \mathrm{~s}$ & $05 \mathrm{~d} 44 \mathrm{~m} 50.0 \mathrm{~s}$ & - & \\
34 & $24 \mathrm{~d} 36 \mathrm{~m} 28.1 \mathrm{~s}$ & $17 \mathrm{~d} 53 \mathrm{~m} 04.0 \mathrm{~s}$ & - & \\
56 & $37 \mathrm{~d} 27 \mathrm{~m} 57.1 \mathrm{~s}$ & $09 \mathrm{~d} 13 \mathrm{~m} 43.2 \mathrm{~s}$ & - & \\
325 & $197 \mathrm{~d} 00 \mathrm{~m} 18.1 \mathrm{~s}$ & $-09 \mathrm{~d} 04 \mathrm{~m} 04.0 \mathrm{~s}$ & - & \\
344 & $218 \mathrm{~d} 31 \mathrm{~m} 05.5 \mathrm{~s}$ & $-15 \mathrm{~d} 00 \mathrm{~m} 08.2 \mathrm{~s}$ & - & \\
392 & $262 \mathrm{~d} 09 \mathrm{~m} 42.5 \mathrm{~s}$ & $-21 \mathrm{~d} 44 \mathrm{~m} 53.0 \mathrm{~s}$ & 6 & star? \\
483 & $343 \mathrm{~d} 38 \mathrm{~m} 37.7 \mathrm{~s}$ & $-08 \mathrm{~d} 57 \mathrm{~m} 26.0 \mathrm{~s}$ & 7 & star? \\
503 & $354 \mathrm{~d} 12 \mathrm{~m} 08.7 \mathrm{~s}$ & $-00 \mathrm{~d} 13 \mathrm{~m} 21.1 \mathrm{~s}$ & - & \\
512 & $357 \mathrm{~d} 40 \mathrm{~m} 02.9 \mathrm{~s}$ & $06 \mathrm{~d} 31 \mathrm{~m} 03.3 \mathrm{~s}$ & - & \\
\hline Mayer & & & & \\
Cat. numb. & RA $(\mathrm{B} 1756.0)$ & Dec $(\mathrm{B} 1756.0)$ & Magnitude & Remarks \\
\hline 41 & $15 \mathrm{~d} 38 \mathrm{~m} 11.1 \mathrm{~s}$ & $05 \mathrm{~d} 49 \mathrm{~m} 04.8 \mathrm{~s}$ & 7 & star? \\
221 & $83 \mathrm{~d} 37 \mathrm{~m} 30.5 \mathrm{~s}$ & $09 \mathrm{~d} 38 \mathrm{~m} 44.9 \mathrm{~s}$ & 8 & \\
231 & $87 \mathrm{~d} 15 \mathrm{~m} 19.7 \mathrm{~s}$ & $17 \mathrm{~d} 47 \mathrm{~m} 35.6 \mathrm{~s}$ & 6.5 & star? \\
\hline \multicolumn{5}{c}{} \\
\hline
\end{tabular}

La Caille's fundamental catalogue (Anonymous (Bailly) 1763, p. Ixv-lxxvij). La Caille used a meridian telescope of Dollond with an achromatic objective for observations of right ascensions, and for declinations his 6-ft sextant carried back from Cape Town. I precessed the positions to J2000.0 and corrected for proper motions from 1761 to 2000 . There are many errors in this catalogue. Of these 515 stars, I had to correct obvious copying or typographical errors for no less than 47 entries. No star could be identified for nine entries (Table 2); some of these entries might correspond to planets (Uranus or minor planets): this deserves further investigation. One entry corresponds to a cluster (Praesepe). For 18 stars the position errors were anomalously large. These problems are certainly due to the incompletion of the project.

The results for the remaining 487 stars are given in Fig. 5 and Table 1 . The systematic error is 1.2 arcsec in right ascension and 3.0 arcsec in declination. There are clear systematic effects both in right ascension errors and declination errors with respect to declination. When corrected for, the rms error in right ascension is reduced to $4.2^{\prime \prime}$ and that in declination $5.6^{\prime \prime}$, which is probably close to the internal errors of the observations. After these corrections, the zodiacal catalogue is slightly better than the fundamental catalogue in right ascension, and slightly poorer in declination.

\section{Mayer's zodiacal catalogue}

At exactly the same time as La Caille, Tobias Mayer (1723-1762) in Göttingen undertook a similar catalogue of zodiacal stars, using a 6-feet radius mural quadrant made by John Bird (1709-1776). The reasons for this duplication are not known: La Caille and Mayer respected each other and exchanged letters. Most of the observations (in general only one per star) were made in 1756 and 1757. Mayer probably reduced his observations himself, and his catalogue was the first relatively accurate zodiacal catalogue ever produced. The observations have been discussed, corrected when possible, reduced again carefully and published by Francis Baily. I used this catalogue (Baily 1831) for my discussion. I randomly selected 200 stars from the 998 stars of the catalogue, corrected one for an obvious typographic error, and could not find any star close to the positions of three entries, again possibly corresponding to planets (Table 2). Two other stars have positions too poor to be included, which left 195 stars. Their positions were precessed to J2000 and 

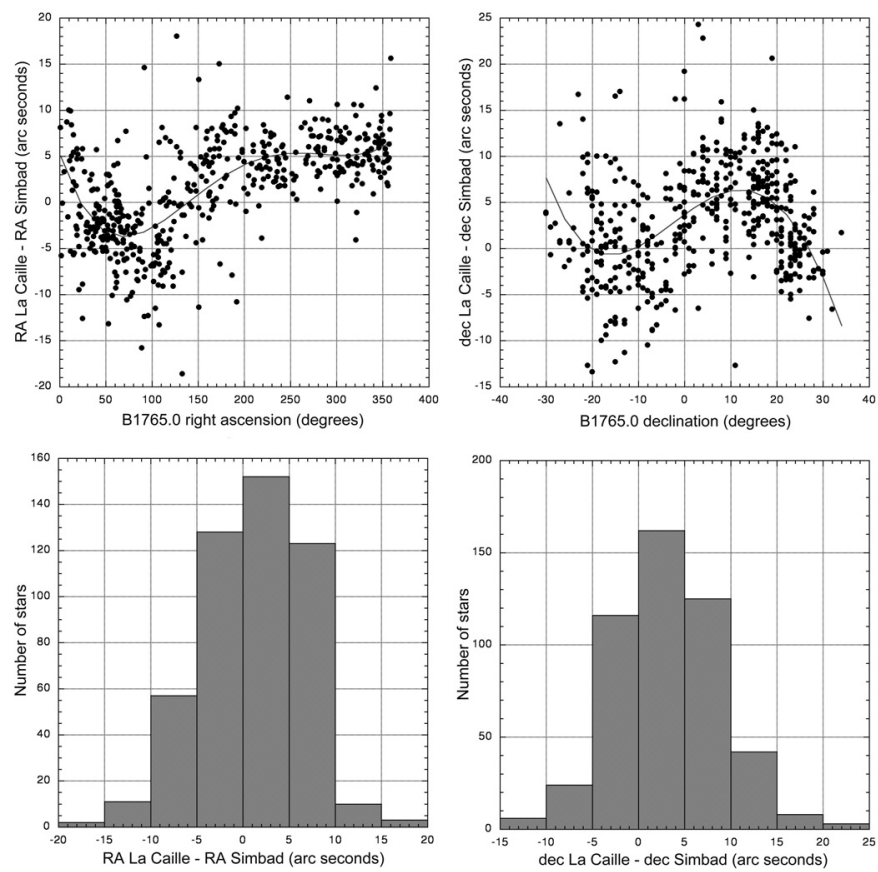

Fig. 5. Results of La Caille's measurements of 487 stars of his zodiacal catalogue. Top left: errors in right ascension as a function of declination, with a fourth-degree polynomial fit of the data. Bottom left: histogram of errors in right ascension. Top right: errors in declination as a function of declination, with a fourth-degree polynomial fit of the data. Bottom right: histogram of errors in declination.

corrected for proper motions in the same way as for the previous catalogues.

The results are given in Fig. 6 and Table 1. There are obvious systematic effects with declination both for errors in right ascension and in declination. Without correcting for these effects, the rms errors are of the order of 5 arcsec in both coordinates, slightly better than those of La Caille's zodiacal catalogue. After correction, they are reduced to 3.7 arcsec rms in right ascension and $3.4 \mathrm{~s}$ in declination.

\section{Bradley's stellar catalogue}

James Bradley (1693-1762) is famous for his discovery of aberration and nutation. From 1750 to his death in 1762, he built a large stellar catalogue, from observations first with an old mural sector and after 1753 with the Bird 8-ft mural sector located in Greenwich, where it can still be seen. This instrument requires to be reversed for observations of stars passing to the north of the zenith. The reductions were made by Friedrich Wilhelm Bessel (1784-1846), as Galle did for Rømer's observations. The catalogue (Bessel 1818) contains 3220 stars. Most of them have been observed several times; for many stars, only one coordinate has been determined. As for the La Caille's southern survey, I chose to work only on a random sample of 215 stars. I precessed the positions to J2000.0 and corrected them for proper motions from 1755 to 2000 . For three stars only the position errors were anomalously large, therefore I excluded them from the sample.

The results for the remaining 212 stars are given in Fig. 7 and Table 1 . There is a systematic error of 1.4 arcsec in right ascension and only 0.3 arcsec in declination, and no obvious systematic error with right ascension and declination. The results are quite good, with an rms of about $3^{\prime \prime}$ in right ascension and $1.8^{\prime \prime}$ in declination.
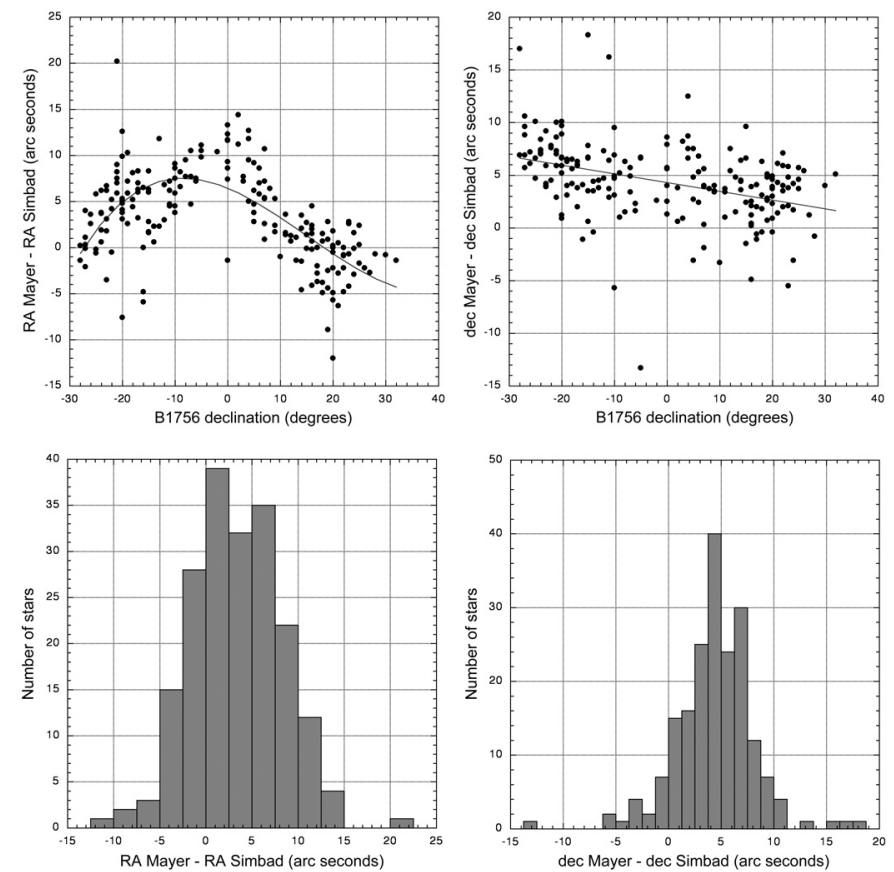

Fig. 6. Results of Mayer's measurements of 195 stars of his zodiacal catalogue. Top left: errors in right ascension as a function of declination, with a third-degree polynomial fit of the data. Bottom left: histogram of errors in right ascension. Top right: errors in declination as a function of declination, with a linear fit to the data. Bottom right: histogram of errors in declination.
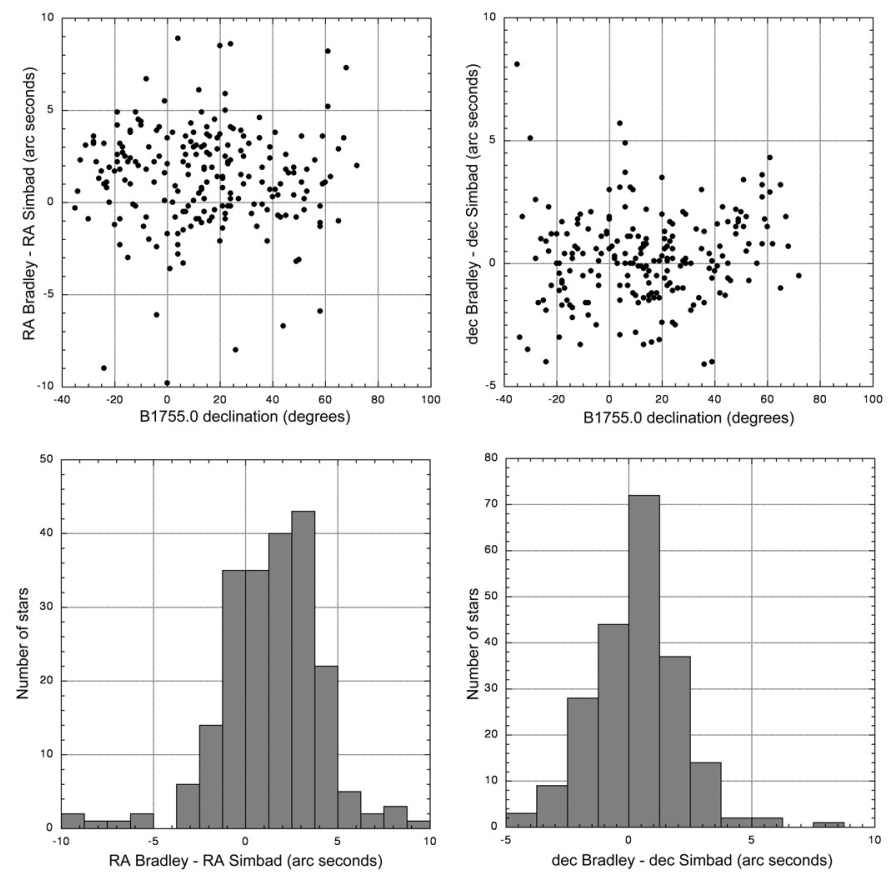

Fig. 7. Results of Bradley's measurements of 194 stars of his zodiacal catalogue. Top left: errors in right ascension as a function of declination, with a third-degree polynomial fit of the data. Bottom left: histogram of errors in right ascension. Top right: errors in declination as a function of declination, with a linear fit of the data. Bottom right: histogram of errors in declination.

\section{Piazzi's stellar catalogue}

Giuseppe Piazzi (1746-1846) built a large catalogue containing 7646 stars from 1792 to 1813, observed in Palermo with 

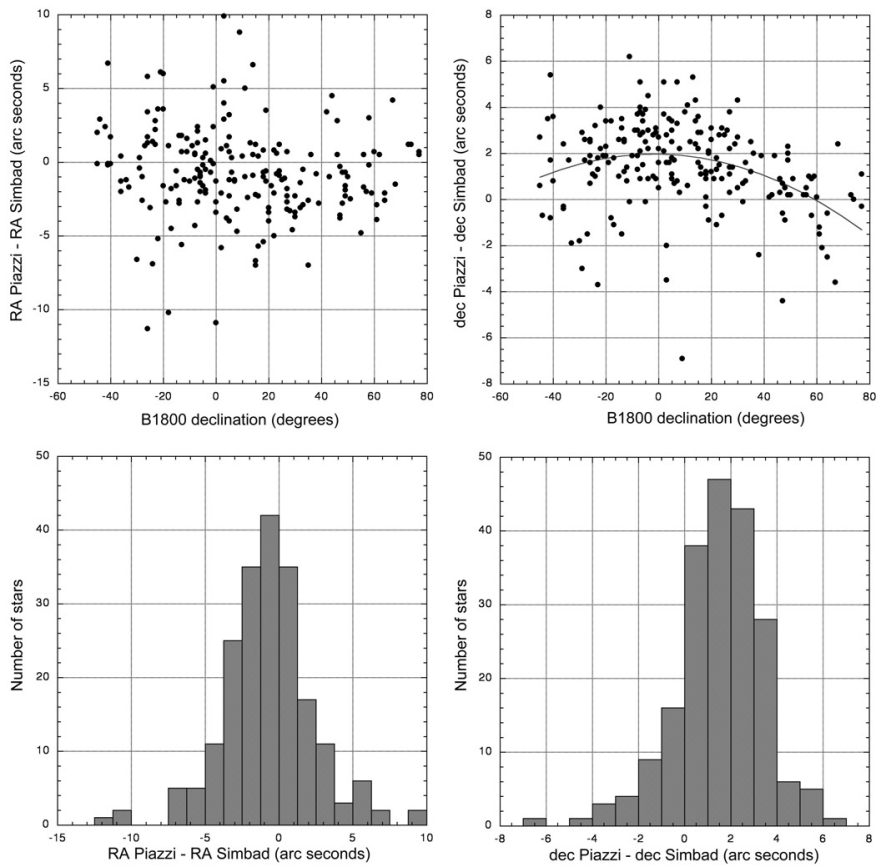

Fig. 8. Results of Piazzi's measurements of 202 stars of his catalogue Top left: errors in right ascension as a function of declination. Bottom left: histogram of errors in right ascension. Top right: errors in declination as a function of declination, with a second-degree polynomial fit of the data. Bottom right: histogram of errors in declination.

an alt-azimuthal circle of Jesse Ramsden (1735-1800). During these observations he discovered the first asteroid, Ceres, on 1 January 1801. His catalogue (Piazzi 1814) was entirely reduced by himself and his collaborators. I randomly selected 203 stars from the catalogue, corrected one typographic error and rejected one star with a too deviant position: the catalogue has obviously be prepared and printed very carefully.

The results for the remaining 202 stars are given in Fig. 8 and Table 1 . They are quite good, with an offset of only -0.7 arcsec in right ascension and $1.5 \mathrm{~s}$ in declination. The rms errors are 3.2 arcsec in right ascension and 2.4 arcsec in declination. There is no obvious systematic deviation in right ascension. In declination, a systematic deviation has been fitted with a seconddegree polynomial, and when corrected for, gives an rms error of 1.8 arcsec.

\section{Lalande's stellar catalogue}

L'Histoire céleste française de Lalande (Lalande 1801), which contains the unreduced observations of approximately 40000 stars, is the first very large stellar catalogue. The measurements have been made essentially by Lalande's "nephew" (actually a cousin) at the observatory of the Military school in Paris with a mural quadrant constructed by Bird in 1774. This huge instrument of 7.5 feet radius is preserved at the Paris Observatory. It required to be reversed with a special device for observations of stars passing to the north of the zenith. The catalogue has been reduced under the supervision of Baily (Lalande $\&$ Taylor 1847). Observations of 3950 more stars passing to the south of the zenith were made between 1801 and 1804 in the same conditions. They were published with careful reductions to B1800.0 by Bossert as a supplement to the main catalogue (Bossert 1892). Bossert corrected a number of typographic or clerical errors. I selected a random sample of 198 entries in this
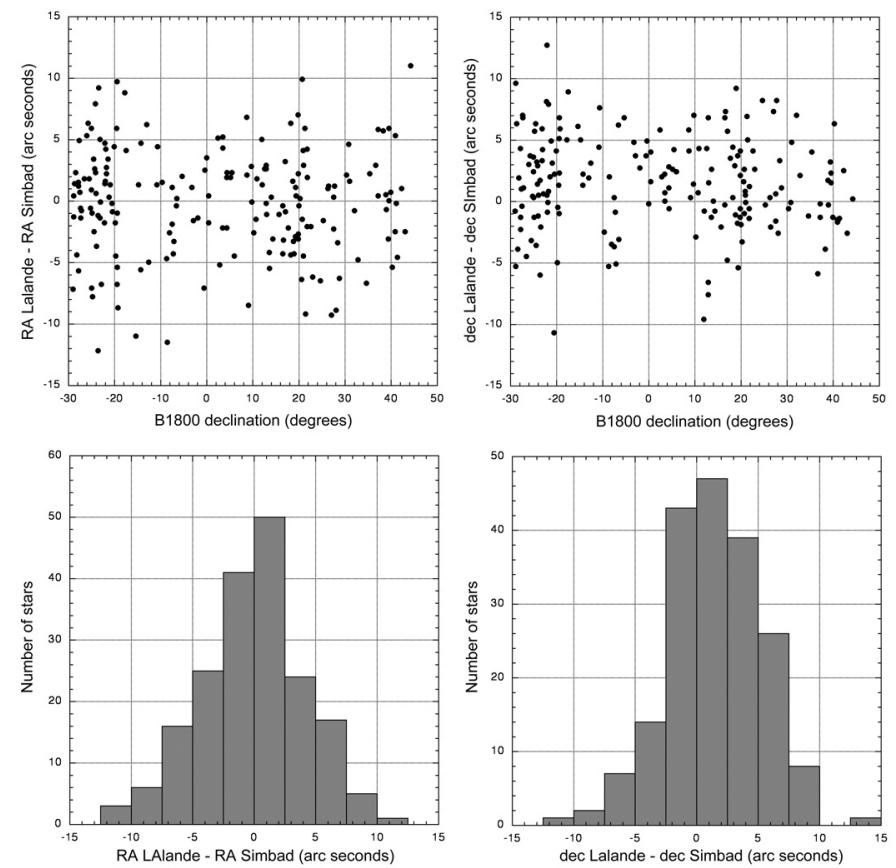

Fig. 9. Results of Lalande's measurements of 188 stars of his supplementary catalogue. Top left: errors in right ascension as a function of declination. Bottom left: histogram of errors in right ascension. Top right: errors in declination as a function of declination. Bottom right: histogram of errors in declination.

Table 3. Magnitude errors in eight 18 th-century catalogues.

\begin{tabular}{lcrcc}
\hline \hline Catalogue & $\begin{array}{c}\text { Number } \\
\text { of stars }\end{array}$ & Mean & rms & St. dev. \\
\hline Flamsteed & 210 & 0.26 & 0.68 & 0.63 \\
La Caille, south. survey & 235 & -0.21 & 0.69 & 0.66 \\
La Caille, fundam. stars & 393 & 0.08 & 0.56 & 0.55 \\
Mayer, zodiacal stars & 172 & 0.61 & 0.93 & 0.70 \\
La Caille, zodiacal stars & 454 & 0.27 & 0.74 & 0.69 \\
Bradley & 215 & 0.41 & 0.67 & 0.54 \\
Piazzi & 202 & 0.48 & 0.74 & 0.56 \\
Lalande & 183 & -0.05 & 0.53 & 0.53 \\
\hline
\end{tabular}

Notes. Errors are given as the catalogue visual magnitudes minus the $V$ magnitudes in Simbad.

supplement. I was unable to find a star corresponding to one entry and rejected nine stars with too deviant positions. This left 188 stars.

The results are given in Fig. 9 and Table 1. There is almost no offset in right ascension, an offset of 1.5 arcsec in declination, and no systematic effect. The rms errors are 4.3 arcsec in right ascension and 4.1 seconds in declination. This is less accurate than the catalogues of Mayer, Bradley and Piazzi. The accuracy of the main catalogue is probably comparable or slightly poorer. In spite of its relatively low accuracy, the Lalande catalogue remained valuable for several decades because of the large number of stars it contains.

\section{Visual magnitudes}

All the studied catalogues contain estimates of the visual magnitudes of the observed stars. For eight of these catalogues, I compared the visual magnitudes with the $V$ magnitudes given in the Simbad database. The results are given in Table 2 and Fig. 9. 

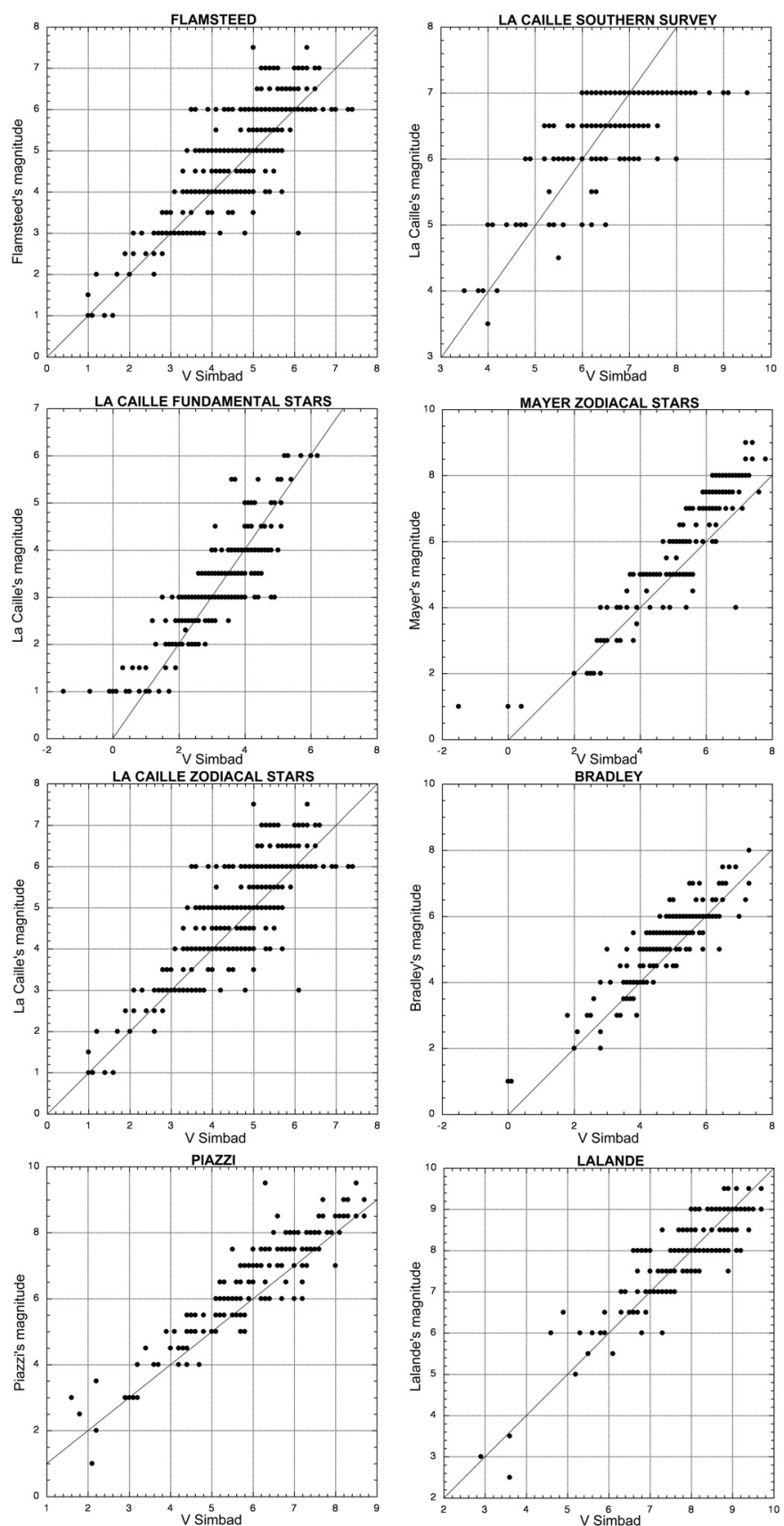

Fig. 10. Comparison of visual magnitudes estimates in eight stellar catalogues with photoelectric $V$ magnitudes from the Simbad database.

Of course, the errors are for a small part due to the variability of the stars. The best estimates are those of La Caille (fundamental stars) and of Lalande.

\section{Conclusion}

This study demonstrates how the improvements of the instruments have affected astrometric accuracy. A first quantitative jump was made possible by Tycho Brahe around 1600, thanks to the excellence of his instruments. Note in passing that most of the instruments used for astrometry in the 17th and 18th centuries derive from Tycho's instruments described in his Astronomice instauratce Mechanica (Tycho Brahe 1602). The adaptation of the astronomical telescope to astrometric instruments caused another quantitative jump. Flamsteed had a

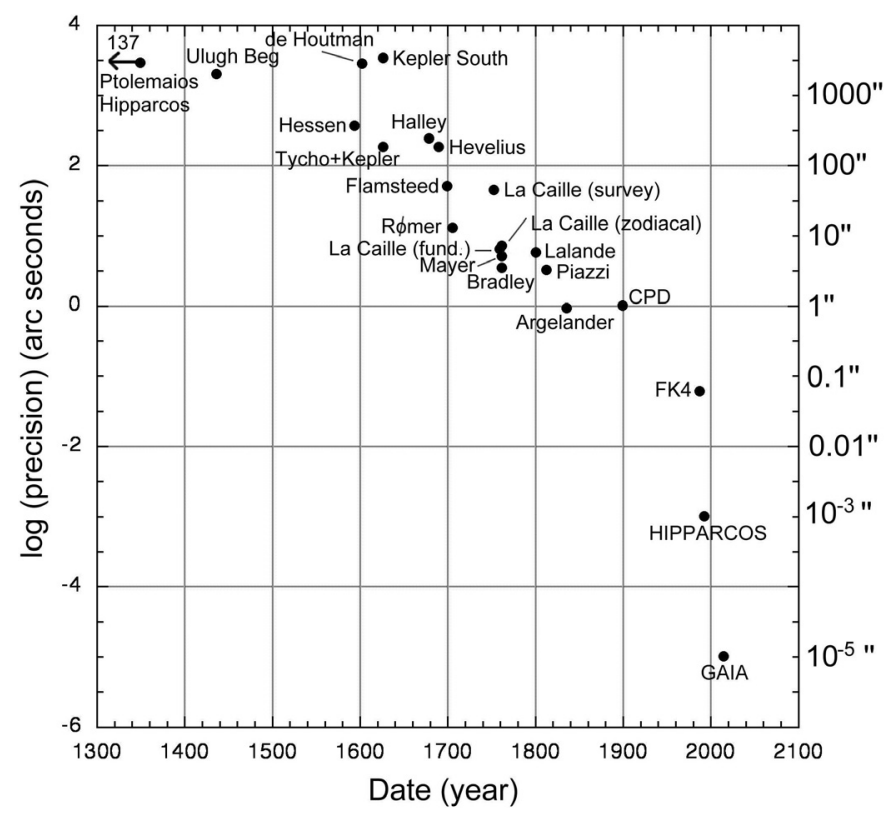

Fig. 11. Accuracy of astrometric catalogues as a function of time. The accuracy is given as $\sqrt{\operatorname{erRA}^{2}+\operatorname{errdec}^{2}}$, where errRA and errdec are the rms error of the catalogue in right ascension and in declination.

telescope adapted to his instruments, with the result that his observations are better than the slightly earlier ones of Hevelius, who observed with the naked eye (Verbunt \& van Gent 2010b). A new generation of instruments was used by astronomers of the 18th century, resulting in another jump in accuracy: the meridian telescope of Rømer and of La Caille's zodiacal observations, the large sextant of La Caille, the mural quadrants of Mayer, Bradley, and Lalande, and the alt-azimuth instrument of Piazzi. The progress in the division of circles was important for these improvements. Whether or not the progress in the astronomical clocks since Huygens played a role is unclear. For example, although the "mercury pendulum", which is insensitive to temperature, was invented in 1726 by George Graham (1673-1751), the clocks used by La Caille did not have temperature-compensating pendulums. After 1750, the reductions included corrections for aberration and nutation, which affected the results of the preceding epochs. An accuracy of between 3 and 6 arcsec was reached in the internal accuracy of the catalogues, some of which were affected by systematic errors of a similar magnitude, however. But this was enough to determine the proper motions of many stars.

A new generation of instruments was required for a further improvement. These were the meridian instruments of the 19th century, which soon allowed an accuracy of the order of 1 arcsec to be reached, with a progressive gain by another order of magnitude during the 20th century. Photography allowed the construction of large catalogues. But the limit of what is possible from the Earth surface was reached, so one had to go to space. The European HIPPARCos satellite allowed us to reach an accuracy better than 0.001 arc second for 100000 stars. The European Gaia satellite will improve this accuracy by a factor 100 for a billion stars.

Figure 11 shows the astrometric accuracy of stellar catalogues as a function of time. This is an rms accuracy defined as $\sqrt{\text { errRA }^{2}+\text { errdec }^{2}}$, where errRA and errdec are the rms error of the catalogue in right ascension and in declination (or in ecliptic longitude and latitude for some early catalogues), uncorrected 
for systematic effects with declination. Figure 11 is an update of Fig. 1 in Høg (2008).

Acknowledgements. All the references listed in the bibliography are available online from ADS, gallica.bnf.fr or Google Books, with the exception of Bessel 1818, Galle 1845, and Glass 2013. I am grateful to the authors of these facilities, and above all to the Centre de Données de Strasbourg (CDS) and to the NASA/IPAC Extragalactic Database (NED).

\section{References}

Anonymous (Bailly) 1763, Ephémérides des mouvemens célestes, pour six années, depuis 1765 jusqu'en 1775, et pour le méridien de la ville de Paris (Paris, Hérissant)

Baily, F. 1831, Mem. Roy. Astron. Soc., 4, 391

Bessel, F. W. 1818, Fundamenta astronomiæ pro anno MDCCLV deducta ex observationibus viri incomparabilis James Bradley in Specula Astronomica Grenovicensi per annos 1750-1762 institutis (Frid Nicolovium, Regiomonti)

Bobis, L., \& Lequeux, J. 2008, J. Astron. History and Heritage, 11, 97

Bossert, J. 1892, Catalogue de 3950 étoiles ramenées à l'équinoxe de 1800,0 (Paris: Gauthier Villars et fils)

Delambre, J.-B. 1827, Histoire de l'Astronomie au dix-huitième siécle, ed. M. Mathieu (Paris: Bachelier)

Flamsteed, J. 1725, Historia Cœlestis Britannicæ (London: Meere)

Galle, J. G. 1845, Olai Roemeri Triduum observationum astronomicarum a MDCCVI, diebus Oct.bXX usque ad XXIII, institutarum reductum et cum tabulis comparatum ab I. G. Galle (Berlin: Typis Academicis)

Glass, I. S. 2013, Nicolas-Louis de la Caille, Astronomer and Geodesist (Oxford University Press); French translation by J. Lequeux as Nicolas-Louis de la Caille, Astronome et Géodesien (EDP Sciences \& Observatoire de Paris)

Gould, B. A. 1890a, AJ, 9, 145
Gould, B. A. 1890b, AJ, 9, 169

Gould, B. A. 1890c, AJ, 10, 1

Gould, B. A. 1890d, AJ, 10, 17

Høg, E. 2008, Astrometric accuracy during the past 2000 years (http: //www . astro.ku.dk/ erik/Accuracy.pdf)

Høg, E. 2009, in 400 years of Astronomical Telescopes, eds. B.Brandl, R. Stuik, \& J. Katgert-Merkelijn, Exp. Astron. (Berlin: Springer), 25, 225

La Caille, N. L. de 1757, Astronomiæ fundamenta novissimis solis et stellarum observationibus stabilita Lutetiæ in Collegio Mazarinæo et in Africa ad Caput Bonæ Spei peractis (Paris: Collombat)

La Caille, N. L. de, Henderson, T., \& Baily, F. 1847, A Catalogue of 9766 Stars in the Southern Hemisphere for the Beginning of the Year 1750 (London: Richard \& John E. Taylor)

Lalande, J. de 1783, Éphémérides des mouvemens célestes pour le méridien de Paris, tome huitième (Paris: Vve Hérissant), 72

Lalande, J. de 1801, Histoire céleste française : contenant les observations faites par plusieurs astronomes français: tome premier (Paris: Imprimerie de la République)

Lalande, J. de, \& Taylor, J. E. 1847, A catalogue of those stars in the Histoire céleste française of Jérôme Delalande: for which tables of reduction to the epoch 1800 have been published by Professor Schumacher... (London: Richard \& John E. Taylor)

Maskelyne, N. 1787, Philosophical Transactions, 77, 151

Piazzi, G. 1814, Principuarum stellarum inerrantium positiones mediæ ineunte sæculo XIX. Ex observationibus habitis in specula Panormitana... (Palermo: ex regia typographia militari)

Tycho Brahe 1602, Astronomiæ instauratæ Mechanica (Nuremberg, L. Hulsium)

Verbunt, F., \& van Gent, R. H. 2010a, A\&A, 516, A28

Verbunt, F., \& van Gent, R. H. 2010b, A\&A, 516, A29

Verbunt, F., \& van Gent, R. H. 2011, A\&A, 530, A93

Verbunt, F., \& van Gent, R. H. 2012, A\&A, 544, A31

Wagman, M. 1987, Journal for the History of Astronomy, 18, 209 